\title{
Effects of a multimodal exercise intervention on physical and cognitive functions in patients with chronic low back pain (MultiMove): study protocol for a randomized controlled trial
}

\author{
Lutz Schega $^{1 \dagger}$, Britta Kaps ${ }^{1 * \dagger}$ (B), Kim-Charline Broscheid ${ }^{1}$, Robert Bielitzki ${ }^{1}$, Martin Behrens ${ }^{1}$, Katharina Meiler ${ }^{2}$,
} Steffen Drange ${ }^{2}$ and Jörg Franke ${ }^{2}$

\begin{abstract}
Background: Chronic low back pain (CLBP) is a common medical condition in adults over the age of 50 . It is associated with severe disability, ranging from physical impairments to psychosocial distress. Since current treatments provide only small to moderate short-term effects, alternative interventions are required, whereby guidelines recommended multimodal approaches. Dancing can be considered as an inherently multimodal approach, as it requires a combination of physical and cognitive functions. Furthermore, it has already been applied effectively in neurorehabilitation. Therefore, it seems promising to merge a dance-therapeutic component together with motor-cognitive, strength and flexibility exercises in a novel multimodal treatment (MultiMove) to target the impaired everyday mobility and cognition of CLBP patients. The aim of this study is to analyse specific physical, cognitive and psychosocial effects of MultiMove in CLBP patients.

Methods: A prospective, two-arm, single-blinded, randomized controlled trial will be conducted with an estimated sample size of 100 CLBP patients, assigned to either the MultiMove group or a control group. The intervention group will receive MultiMove twice a week for 60 min each over a period of 12 weeks. The primary outcome will be the mobility and function of the lower extremities assessed by the Timed Up-and-Go Test. Secondary outcomes comprise further physical and physiological functions (e.g. gait variability and haemodynamic response in the prefrontal cortex during motor-cognitive dual tasks), subjective health state (e.g. disability in daily life), executive functions (e.g. cognitive flexibility) and psychosocial aspects (e.g. kinesiophobia). Measures will be taken at baseline, after the intervention and at a 12-week follow-up. It is assumed that MultiMove improves the mentioned outcome parameters.

(Continued on next page)
\end{abstract}

\footnotetext{
*Correspondence: britta.kaps@ovgu.de

†Lutz Schega and Britta Kaps have contributed equally to this manuscript and share the first authorship

'Health and Physical Activity, Department of Sport Science, Institute III, Otto von Guericke University Magdeburg, Zschokkestraße 32, 39104 Magdeburg, Germany

Full list of author information is available at the end of the article
}

(c) The Author(s). 2021 Open Access This article is licensed under a Creative Commons Attribution 4.0 International License, which permits use, sharing, adaptation, distribution and reproduction in any medium or format, as long as you give appropriate credit to the original author(s) and the source, provide a link to the Creative Commons licence, and indicate if changes were made. The images or other third party material in this article are included in the article's Creative Commons licence, unless indicated otherwise in a credit line to the material. If material is not included in the article's Creative Commons licence and your intended use is not permitted by statutory regulation or exceeds the permitted use, you will need to obtain permission directly from the copyright holder. To view a copy of this licence, visit http://creativecommons.org/licenses/by/4.0/ The Creative Commons Public Domain Dedication waiver (http://creativecommons.org/publicdomain/zero/1.0/) applies to the data made available in this article, unless otherwise stated in a credit line to the data. 


\begin{abstract}
(Continued from previous page)
Discussion: The combined assessment of changes in physical and cognitive functions as well as neuropsychological aspects in response to MultiMove will allow a better understanding of the motor-cognitive adaptations induced by multimodal exercises in CLBP patients. The specific conclusions will lead to recommendations for the conservative treatment approach in this clinically relevant patient group.

Trial registration: German Clinical Trial Register (ID: DRKS00021696 / 10.07.2020), https://www.drks.de/drks_web/ navigate.do?navigationld=trial.HTML\&TRIAL_ID=DRKS00021696
\end{abstract}

Keywords: Motor control, Dance, Gait variability, Dual task, Functional near-infrared spectroscopy

\section{Background}

Low back pain (LBP) is a frequent medical condition and major economic health issue in Germany with a lifetime prevalence of around $85 \%$ in the population [1]. In a study considering the global burden of diseases, LBP was classified as the condition highest in terms of disability. Furthermore, it was shown that the prevalence increases with age and that the chronic type of LBP (CLBP, with and without leg pain) was associated with the most severe disability [2]. Thereby, CLBP is defined as pain in the lumbar region of the spine lasting for at least 3 months [3]. Although, only a small percentage of LBP patients develop CLBP [3, 4], they are responsible for the majority of costs associated with LBP caused by recurrent health care consultations, occupational incapacity and early retirement [5-8]. Degenerative changes in the lumbar spine are strongly associated with CLBP $[5,9]$. However, in most of the cases they cannot sufficiently explain the underlying pain mechanisms and the related impairments [4, 10-12].

On a physical level, CLBP patients can experience limitations such as insufficient muscular trunk stabilisation and strength [13], as well as a poor static postural control and an altered gait performance [14-17]. Moreover, research indicates that motor-cognitive dual task performance (e.g. walking while performing a cognitive task) is reduced in CLBP patients [14, 18]. This could be explained by the fact that pain can interfere with gait control, e.g. executive functioning $[14,19]$. This is consistent with the observation that patients with chronic pain showed reduced cognitive functioning (e.g. executive functions) [20]. These motor-cognitive deficits lead to limitations in daily activities and may provoke an increased risk of falling $[13,19,21]$. Nevertheless, it is essential to consider that also psychological factors like anxiety, depression and/or passive coping strategies affect the development and maintenance of CLBP [4, 22]. In addition, these impairments reinforce each other: e.g. the fear of pain leads to a decreased daily activity level (described as kinesiophobia [23]), contributing to the insufficient muscular stabilisation and disability level [16, 24]. These multidimensional negative experiences impair the patients beyond the chronic pain and contribute to a reduction in health-related quality of life $[25,26]$.

To address the multifaceted impairments of CLBP, multidisciplinary approaches are recommended [3]. They have shown superior effectiveness in decreasing pain and disability compared to usual care $[27,28]$. In this regard, a combination of conservative (e.g. pharmacological, physical, psychological) interventions are applied by specialists of diverse professions [16, 27, 29]. Building on this holistic treatment approach and to further improve the effectiveness, we have designed a novel multimodal exercise intervention (MultiMove) for CLBP patients. MultiMove addresses the described impaired physical (e.g. dynamic postural control, trunk stability), cognitive (e.g. executive functions) and psychological (e.g. kinesiophobia) domains of CLBP, through the combination of three training components.

The basic component of MultiMove consists of a strength and flexibility training, as they have been shown to be the most effective exercise interventions for CLBP patients, so far [30, 31]. To moreover address the impaired dual task performance and the executive functions of CLBP patients, the second component of MultiMove is a motor-cognitive training according to the Life Kinetik ${ }^{\circ}$ concept [32]. In healthy older adults, this kind of training has already been shown to decrease gait variability as well as reduce fear of falling [33, 34]. Because balance/stabilisation training has been shown to be advantageous for CLBP patients [30], the third component comprises a dynamic balance training using dance-therapeutic elements. Moreover, dancetherapeutic exercises inherently require a combination of motor and cognitive functions (e.g. to remember and execute several partial movements at the same time) and are further associated with social interaction [35, 36]. In combination with the beneficial effects of the motor-cognitive training, this social interaction might help to overcome the kinesiophobia. In line with this, it has been shown that dance interventions improve functional mobility, sensorimotor and endurance performance in healthy adults and elderly [37-39]. Besides, dance interventions might lower gait 
variability and improve gait speed [40, 41]. Additionally, it has been revealed that dancing is superior to conventional training interventions (involving mainly repetitive physical exercises) with regard to the training-induced brain plasticity in elderly people [42]. Due to these multiple benefits, dance-therapeutic interventions have already been effectively applied in the field of neurological rehabilitation [36, 43-46]. Therefore, extending the approach to the needs of CLBP patients seems promising.

With the combination of these three components, MultiMove is expected to contribute to an improvement in physical and physiological functions (e.g. Timed Upand-Go [TUG] performance, gait variability, haemodynamic response in the prefrontal cortex [PFC]) as well as cognitive performance [aspects of executive functions]). A reduction in pain as well as an increase in quality of life are assumed to come along with those changes.

\section{Primary objective}

1) To test the effect of MultiMove on CLBP patients' physical function (TUG performance) relative to the conventional conservative therapy.

\section{Secondary objectives}

1) To examine changes in the LBP specific disability (Oswestry Disability Index, ODI) and health-related quality of life (EQ-5D-5L) between groups and over time.

2) To evaluate the specific physical changes induced by MultiMove (gait variability, flexibility, functional exercise capacity and leg extensor muscle power).

3) To analyse if MultiMove induces changes in selected executive functions (inhibitory control, cognitive flexibility) and/or in the haemodynamic response in the PFC during dual task standing and walking in CLBP patients.

4) To determine the potential long-term psychosocial effects (Kinesiophobia, pain coping strategies) provoked by MultiMove.

\section{Methods}

\section{Study design and setting}

The current study is designed as a prospective, twoarm randomized, controlled, superiority clinical trial. To assess the effect of MultiMove on the measures presented above in CLBP patients, subjects will be allocated to an intervention group (IG) or a control group (CG) using block randomization (1:1 allocation) [47]. The primary and secondary outcomes will be assessed at three time points (see Fig. 1, a detailed overview of the outcome measures can be found in Table 3).

The first measurements will be conducted directly before intervention onset (baseline), the second directly after the intervention (post, week 12-14, t1), and the third after additional 12 weeks (follow-up, week 24-26, t2). All investigators will be blinded to group allocation. Data collection will be performed at the Otto von Guericke University (OvGU) Magdeburg (Germany). The protocol is in line with the principles of the Declaration of Helsinki and was approved by the institutional review board (IRB) of the OvGU (registration number: 182/18).

\section{Study population and eligibility criteria}

MultiMove is designed for outpatients with CLBP associated with degenerative diseases of the lumbar spine and aged $\geq 50$. They will be recruited by the orthopaedic department of the Klinikum Magdeburg gGmbH in collaboration with local orthopaedist. Information will be provided in written form in doctor's practices as well as personally in their consultations. In addition, the study will be advertised via local newspaper.

To be considered for the study, participants must comply all off the following inclusion criteria:

(i) age $\geq 50$ years

(ii) an average low back pain over the last 4 weeks $\geq 4$ on a numerical rating scale (NRS) from 0 - no pain to 10 - pain as bad as it could be

(iii) duration of LBP and/or neurogenic claudication $\geq 3$ months

(iv) International Classification of Diseases 10th revision diagnosis code related to chronic back pain (ICD10: M54 Dorsalgia; M48.0 Spinal stenosis; M54.5 Low back pain; M54.4 Lumbago with sciatica; M54.1 Radiculopathy; M41.5 Other secondary scoliosis; M43.1 Spondylolisthesis; M42.1 Adult osteochondrosis of spine; M51.2 Other specified intervertebral disc displacement; M47.8 other spondylosis; M53.2 Spinal instabilities)

Exclusion criteria are: (i) more than two prior operations of the spine, (ii) spinal fusion of more than three segments, (iii) any spinal operation within the last 6 months, (iv) dependence on a walking aid or inability to walk more than $300 \mathrm{~m}$ at a stretch, (v) reduction in strength of more than $25 \%$ according to Janda [48] (level 0-3), (vi) congenital spine deformities, (viii) any neurological, cardiovascular, psychological and musculoskeletal diseases that preclude the execution of the intervention and the measurements. 


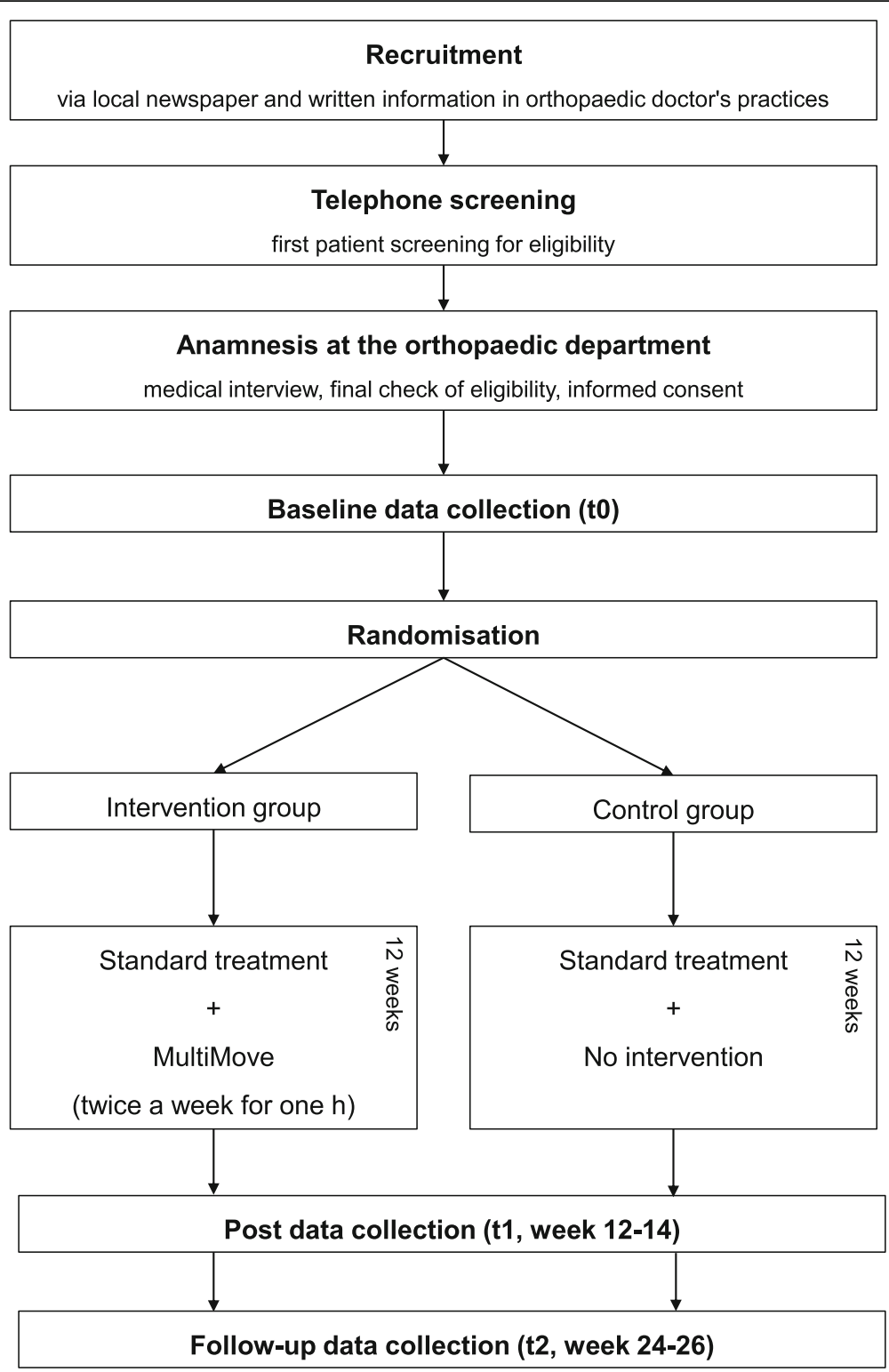

Fig. 1 Study schedule of enrolment, intervention and assessments

\section{Sample size}

Sample size was calculated using G*Power (version 3.1.9.7.). Because previous studies have shown large effect sizes for changes in the TUG performance after single interventions (dancing [49], strength training [50]) as well as after multimodal interventions [51] in healthy older adults, a large effect size $(f=0.40)$ was assumed for the sample size calculation. Considering an $\alpha$ level of 0.05 , a power of 0.95 , two groups and two covariates (baseline scores and, when required, e.g. age, sex, etc.), the required total sample size for an analysis of covariance (ANCOVA) amounted 84 participants. With a hypothesized dropout rate of around 15\%, a total sample size of 100 participants seems to be legitimated.

\section{Study interventions}

Both groups (IG and CG) will receive the standard conservative therapy comprising physiotherapy and drugbased pain management according to their individual needs. Additionally, the participants of the IG will receive MultiMove twice a week on non-consecutive days over a period of 12 weeks. The duration of each session is set to $60 \mathrm{~min}$ (see Table 1) with a successive progression in the level of difficulty over time (see Table 2). Each session consists of motor-cognitive, dancing and strength or flexibility exercises with reference to the current recommendations for physical activity in older adults provided by the American College of Sports Medicine [52]. 
Table 1 Time slots for weekly training sessions (WTS) of $2 \times 60$ min in all phases

\begin{tabular}{lll}
\hline Training components & Phase 1-3 \\
\cline { 2 - 3 } & WTS 1* (Time) & WTS 2* (Time) \\
\hline Life Kinetik $^{\oplus}$ (including warm-up) & $25 \mathrm{~min}$ & $25 \mathrm{~min}$ \\
Dance & $25 \mathrm{~min}$ & $25 \mathrm{~min}$ \\
Strength & - & $10 \mathrm{~min}$ \\
Flexibility & $10 \mathrm{~min}$ & - \\
\hline
\end{tabular}

${ }^{*} \geq 48 \mathrm{~h}$ rest between WTS

The classes will start with the motor-cognitive component, which includes a warm-up phase with movements for all major muscle groups. The following motor-cognitive exercises are in line with a licensed concept (Life Kinetik ${ }^{\circ}$, [32]). Here, motor and cognitive tasks have to be performed simultaneously, requiring a redistribution of attentional resources. The exercises are designed in a way that they can hardly be executed without mistakes and the difficulty level will be adapted to the ability of the participants. During the following dance part, the participants will learn choreographies with increasing complexity over time, which will be adapted to their disability level. The choreographies will include different dance styles (e.g. Latin, Standard, Jazz) whereby each style focuses on a specific aspect, e.g. spatial orientation in Line dance or posture in Latin dance. To ensure that on the one hand different performance levels do not influence the dancing, but on the other hand, participants interact socially, the choreographies will be performed individually in a group setting and will be supplemented by dance formations. The final strength and flexibility training will focus on evidence-based exercises for the spine, back, abdominal and hip muscles [53].

The sessions will be supervised by two accredited instructors and will be conducted with a maximum of 15 participants. Instructors will keep an attendance list at each class. If participants are absent for two consecutive intervention sessions, they will be contacted by telephone. In order to improve attendance to MultiMove, participants of the IG will be asked to visit at least $80 \%$ of the classes. Moreover, participants will receive global information about the purpose and usefulness of the intervention at the first training session as well as specific explanations during the respective exercises. The individual perceived enjoyment of MultiMove will be assessed via the German version of the Physical Activity Enjoyment Scale once a week [54].

Additionally, instructors will keep an activity log in each class to maintain intervention fidelity. Activity log will include a check-list with essential components of the intervention protocol and will be reviewed by an independent assessor. If participants will not be able to perform the planned exercises, because of the physical requirements, small individually adjusted modifications will be made and protocolled. Furthermore, if participants should premature terminate out of MultiMove, reasons will be recorded (e.g. personal reasons, relocation, illness that prevent physical activity or death).

The participants of the CG will be examined in the same time period as the IG receiving only the standard therapy (physiotherapy and drug-based pain management according to their individual needs), with the possibility to attend MultiMove as well, but not before the follow-up data collection is completed.

Besides, both groups will be asked not to start any (further) specific training intervention or pain treatment during the study period. Their physical activity level will be monitored by a questionnaire.

\section{Outcome measures}

A detailed description of the outcome measurements is following, whereby Table 3 presents an overview of the addressed constructs with the assessment methods and respective parameters. Moreover, the measurement time points are marked in Table 3.

Table 2 Details of MultiMove with exercise examples

\begin{tabular}{|c|c|c|c|c|}
\hline \multirow[t]{2}{*}{$\begin{array}{l}\text { Training } \\
\text { components }\end{array}$} & \multirow[t]{2}{*}{ Exercise } & $\begin{array}{l}\text { Examples of exercise } \\
\text { Phase } 1\end{array}$ & \multirow{2}{*}{$\begin{array}{l}\text { Examples of exercise } \\
\text { Phase } 2 \\
\text { Week 5-8 | Intermediate }\end{array}$} & \multirow{2}{*}{$\begin{array}{l}\text { Examples of exercise } \\
\text { Phase } 3 \\
\text { Week 9-12 | Advanced }\end{array}$} \\
\hline & & Week 1-4 | Foundational & & \\
\hline Life Kinetik $^{\oplus}$ & cognitive-motor exercises & $\begin{array}{l}\text { balancing while } \\
\text { counting backwards }\end{array}$ & $\begin{array}{l}\text { bouncing a ball while solving } \\
\text { arithmetic tasks }\end{array}$ & $\begin{array}{l}\text { ball juggling and reacting to } \\
\text { external stimuli }\end{array}$ \\
\hline Dance & $\begin{array}{l}\text { choreographies focusing on different } \\
\text { dance styles }\end{array}$ & Line dance, Irish dance & Pasodoble, Salsa, Cha-cha-cha & Tango, Salsa \\
\hline Strength & core stability and strengthening & $\begin{array}{l}\text { pelvic tilt, abdominal } \\
\text { hollowing }\end{array}$ & curl up, bridge, bird dog & $\begin{array}{l}\text { side plank, hip abductor wall } \\
\text { squat }\end{array}$ \\
\hline Flexibility & $\begin{array}{l}\text { range of motion through static } \\
\text { stretching of core and hip muscles }\end{array}$ & $\begin{array}{l}\text { knee to chest, } \\
\text { piriformis stretch }\end{array}$ & $\begin{array}{l}\text { hamstring stretch, hip flexor } \\
\text { stretch, seated flexion }\end{array}$ & $\begin{array}{l}\text { warrior one pose, warrior } \\
\text { two pose }\end{array}$ \\
\hline
\end{tabular}


Table 3 Constructs, assessment methods, parameters and data collection schedule

\begin{tabular}{|c|c|c|c|c|c|}
\hline Constructs & Assessment methods & Parameters & $\begin{array}{l}\text { Baseline } \\
\text { (t0) }\end{array}$ & $\begin{array}{l}\text { Post } \\
\text { (t1) }\end{array}$ & $\begin{array}{l}\text { Follow- } \\
\text { up }(\mathrm{t} 2)\end{array}$ \\
\hline \multicolumn{6}{|l|}{ Physical functions } \\
\hline $\begin{array}{l}\text { Mobility and function of the lower } \\
\text { extremities }\end{array}$ & Timed Up-and-Go Test ${ }^{1}$ & Time (s) to complete the task & $x$ & $x$ & $x$ \\
\hline $\begin{array}{l}\text { Functional exercise capacity and } \\
\text { dynamic postural control }\end{array}$ & $\begin{array}{l}\text { Six-minute walk test with inertial } \\
\text { measurement units (IMUs) and an } \\
\text { electro-cardiogram }\end{array}$ & $\begin{array}{l}\text { Walking distance }(\mathrm{m}) \text { in } 6 \text { min, gait } \\
\text { kinematics (e.g. minimum toe } \\
\text { clearance, gait variability) and heart } \\
\text { rate }\end{array}$ & $x$ & $x$ & $x$ \\
\hline $\begin{array}{l}\text { Functional leg extensor muscle } \\
\text { power }\end{array}$ & $\begin{array}{l}\text { Five-repetition sit-to-stand test on a } \\
\text { force plate }\end{array}$ & $\begin{array}{l}\text { Time (s) and rate of force } \\
\text { development }\end{array}$ & $x$ & $x$ & $x$ \\
\hline Flexibility & $\begin{array}{l}\text { Instrumented trunk range of motion } \\
\text { assessment }\end{array}$ & Active range of motion $\left(^{\circ}\right)$ & $x$ & $x$ & $x$ \\
\hline \multicolumn{6}{|l|}{ Subjective health state } \\
\hline $\begin{array}{l}\text { Back pain associated disability in } \\
\text { daily life }\end{array}$ & Oswestry Disability Index & Total score (0-100\%) & $x$ & $x$ & $x$ \\
\hline Health-related quality of life & EQ-5D-5L & $\begin{array}{l}\text { Index value }(-0.66 \text { to } 1) \text { and subjective } \\
\text { overall health state }(0-100)\end{array}$ & $x$ & $x$ & $x$ \\
\hline \multicolumn{6}{|l|}{ Motor-cognitive dual task performance } \\
\hline $\begin{array}{l}\text { Static and dynamic postural control } \\
\text { during a cognitive challenge, } \\
\text { haemodynamic response in the } \\
\text { prefrontal cortex (PFC) and dual task } \\
\text { costs }\end{array}$ & $\begin{array}{l}\text { Single and dual task bipedal stance } \\
\text { performance on a force plate (with and } \\
\text { without closed eyes) as well as gait } \\
\text { analysis with IMUs, functional near- } \\
\text { infrared spectroscopy and finger } \\
\text { oximeter }\end{array}$ & $\begin{array}{l}\text { Deviation of the center of pressure } \\
\text { (e.g. sway and velocity), gait kinematics } \\
\text { (e.g. minimum toe clearance, gait } \\
\text { variability, gait velocity), oxy- and } \\
\text { deoxyhaemoglobin concentrations in } \\
\text { the PFC, number of correct } \\
\text { calculations and peripheral } \\
\text { oxygenation }\end{array}$ & $x$ & $x$ & $x$ \\
\hline \multicolumn{6}{|l|}{ Executive functions } \\
\hline Inhibitory control & Colour-Word-Interference Test & Time (s) and error rate & $x$ & $x$ & $x$ \\
\hline Cognitive flexibility & Trail Making Test & Time (s) and error rate & $x$ & $x$ & $x$ \\
\hline \multicolumn{6}{|l|}{ Psychosocial aspects } \\
\hline Fear of movement & Tampa Scale of Kinesiophobia & Total score (11-44) & $x$ & $x$ & $x$ \\
\hline Pain coping strategies & Coping Strategies Questionnaire & $\begin{array}{l}\text { Total score for each coping strategy } \\
(0-36)\end{array}$ & $x$ & $x$ & $x$ \\
\hline \multicolumn{6}{|l|}{ Additional } \\
\hline Sociodemographic data & Intake form & e.g. age, sex, education & $x$ & & \\
\hline Physical characteristics & Intake form & e.g. height, weight & $x$ & $x$ & $x$ \\
\hline Current pain intensity & Numerical rating scale & Score $(0-10)$ & $x$ & $x$ & $x$ \\
\hline $\begin{array}{l}\text { Specific characteristic and treatment } \\
\text { history of chronic pain }\end{array}$ & German Pain Questionnaire & $\begin{array}{l}\text { Descriptive data, time period (months) } \\
\text { of chronic pain, pain intensity }(0-10) \text { in } \\
\text { the last } 4 \text { weeks }\end{array}$ & $x$ & $x$ & $x$ \\
\hline Level of everyday activity & $\begin{array}{l}\text { Freiburger Questionnaire on Physical } \\
\text { Activity }\end{array}$ & Time (h) of physical activity in a week & $x$ & $x$ & $x$ \\
\hline State fatigue & $\begin{array}{l}\text { Fatigue subscale of the Profile of Mood } \\
\text { States }\end{array}$ & Score $(0-42)$ & $x$ & $x$ & $x$ \\
\hline Depression & Beck Depression Inventory II & Total score (0-63) & $x$ & $x$ & $x$ \\
\hline
\end{tabular}

${ }^{1}$ Primary outcome measure; all instruments will be presented in German 


\section{Primary outcome measure Timed Up-and-Go Test}

The TUG Test is commonly used in geriatric settings to assess function of the lower extremities, mobility and fall risk [55]. The patient will sit on a standard arm chair with his back and arms resting on it. A line will be marked on the floor $3 \mathrm{~m}$ away from the chair. At the command "go" the patient has to get up (without using the arms), walk as quickly and stable as possible to the marked line, turn $180^{\circ}$, return to the chair and get back into the starting position $[55,56]$. To ensure, that the test procedure has been understood, the procedure can be tried once before the actual test. The time needed from releasing the back from the backrest to touching it again will be measured during the test. The TUG will be performed two times, whereby the fastest trial will be considered. It has been shown, that the TUG test can be performed reliably $[55,57]$.

\section{Secondary outcome measures Physical functions}

Six-minute walk test The Six-minute walk test (6MWT) is an objective measurement to assess the functional exercise capacity close to activities of daily living by measuring the total walking distance accomplished in 6 min [58]. During the 6MWT, the participants will walk back and forth on a $15 \mathrm{~m}$ long track as fast as they can for $6 \mathrm{~min}$. Additionally to the standard protocol, gait kinematics (e.g. minimum toe clearance (MTC) [59], double step length (DSL), gait velocity [60] and their variability) will be recorded, using three inertial measurement units (IMUs, one at each foot and one at the sternum, MTw, Xsens Technologies B.V., Netherlands). The calculation of gait parameters will be in line with the protocol of Hamacher et al. [59]. Furthermore, the participants will wear a portable 3-channel electrocardiogram with a finger oximeter (SOMNOtouch ${ }^{\text {тM }}$ NIBP; SOMNOmedics GMbH Germany) to assess heart rate and peripheral oxygenation as an objective measurement of exercise intensity. Additionally, the subjective level of exhaustion [61] as well as the current pain situation on a NRS from 0 (not fatigued at all / no pain) to 10 (total fatigue \& exhaustion / pain as bad as it could be) will be enquired before and after the 6MWT.

Five-repetition sit-to-stand test The leg extensor muscle power will be assessed with the fiverepetition sit-to-stand test (FRSTST) [62, 63] performed on a force plate (Type 9260AA, Kistler Group, Winterthur, Switzerland; sampling frequency: $1000 \mathrm{~Hz}$ ). The participants will sit on a standard chair without armrest in front of a force plate. They will be asked to fold their arms across their chests, place their feet on the plate and to stand up and sit down as fast as possible five times in a row [62, 63]. During the performance, the investigator will ensure that the participants getting up fully upright and touch the surface of the seat completely. Participants are allowed to practice the process two times. The total time, from the starting signal to the fifth time seating, and the force-time curves, from which rate of force development can be derived, will be measured.

Instrumented trunk range of motion assessment $\mathrm{A}$ sensor-based measuring device (mobbe ${ }^{\oplus}$ med, SportMed A.G. SA, Luxembourg) will be used to assess active trunk range of motion. Specifically, the participants will be asked to perform extension-flexion, lateral flexion (left and right) and rotation (left and right) of the spine. Thereby, the range of motion as well as the subjective pain sensation rated on a NRS (0-10) will be recorded.

\section{Subjective health state}

The Oswestry disability index The ODI is a conditionspecific, self-administered questionnaire for spinal disorders $[64,65]$. It consists of 10 items, scored on a 6-point scale (0 to 5 ). One item assesses the extent of back pain, while the other nine ask for the difficulties in different activities of daily life because of the pain: personal care, lifting, walking, sitting, standing, sleeping, sex life, social life and travelling [66]. All items refer to the current ("today") pain situation. The total score is multiplied by 2 and is presented as a percentage, whereby a higher score represents a higher level of disability [66]. In this study, the reliable and valid German version of the ODI will be used [66].

EQ-5D-5L The EQ-5D-5L has been developed by the EuroQol Group and is an international, standardized, short questionnaire to assess generic health-related quality of life [67]. In the current study the German selfcomplete paper version will be used, which consists of two parts. The first part includes five items (health dimensions: mobility, self-care, usual activities, pain/discomfort and anxiety/depression). Here, each dimension can be scored on a 5-point scale (severity levels) corresponding to the problems the respondent has in this area $(0=$ no problem, $5=$ extreme problems). The second part consists of a visual analogue scale (VAS) ranging from 0 to 100 (worst to best health the respondent can imagine), on which participants should indicate their current subjective overall health state. To calculate a EQ-5D-5L summary index score a country specific value set, which weights each level in each dimension specifically, will be used [68]. 


\section{Motor-cognitive dual task performance}

Single and dual task postural control performance with functional near-infrared spectroscopy and inertial measurement units A portable functional nearinfrared spectroscopy (fNIRS/NIRSport, NIRx Medical Technologies, NY, USA) and IMUs (described above) will be applied while performing postural control tasks with and without a cognitive task. Due to the fNIRS system requirements, the testing protocol has to alternate constantly between baseline (standing position with eyes open) and one of four action tasks. First two dynamic postural control tasks are conducted in random order: single task walking (STW) or dual task walking (DTW). Subsequently, two static postural control tasks - dual task standing (DTS) or closed eyes standing (CES) - are executed in a random order. For the dynamic conditions, the participants will have to walk a $15 \mathrm{~m}$ track back and forth with their individual comfort velocity. During static conditions, participants will stand as stable as possible on a force plate (Type 9260AA, Kistler Group, Winterthur, Switzerland; sampling frequency: $100 \mathrm{~Hz}$ ) in an upright bipedal position with arms akimbo, looking straight ahead. The positions of the feet will be measured and noted, to ensure reliable measurements. In the dual task conditions, the participants will count backwards in steps of three from a pre-defined 3-digit number (between 300 and 400) while walking (DTW) or standing (DTS). Here, participants will be instructed to pay equal attention to both tasks. During the CES participants will be asked to close their eyes while continuing the stable stand.

Each action part will be assessed over a total of 2 min (split in four times $30 \mathrm{~s}$ ) and the baseline over 2: 45 min (five times $33 \mathrm{~s}$ ). Hence, the total measuring time will be $4: 45$ min per task. In order to familiarize the participants with each task, a short version (10 s: $10 \mathrm{~s})$ will be performed once before actual testing. During all four tasks, the haemodynamic response in the PFC (relative oxy- and deoxyhaemoglobin concentrations) will be measured by a fNIRS system. Data recording, processing and analyses will be in line with the recently published guidelines for fNIRS in posture and gait research [69]. Additionally, gait kinematics (e.g. MTC, DSL, gait velocity and their variability) will be recorded with IMUs during the dynamic postural exercises. During the static tasks the deviation of the centre of pressure (e.g. sway and velocity) will be assessed based on the force plate data. The error rate and number of correct calculations of the cognitive task will be captured with a voice recorder. Furthermore, the participants will wear the SOMNOtouch (described above) to check for confounders in the haemodynamic response [70]. Here the outcome parameters are: heart rate, heart rate variability indices and peripheral finger oxygenation.

\section{Executive functions}

Colour-Word-Interference test The Colour-WordInterference Test (based on the Stroop effect [71]) is a frequently used neuropsychological test to assess inhibitory control [72, 73]. Here, the participants have to read out loud and as fast as possible nine tables. These tables consist of three different consecutive tasks that are presented three times: (i) read colour names (printed in black ink), (ii) name the colour of colour bars, (iii) name ink colour of colour names (printed colour never matches the written colour name) [71, 73]. The (iii) task is the incongruent condition requiring to inhibit the more automated task (e.g. reading the colour name), which is called the Stroop effect [71, 73]. The required time is stopped individually for each table and the corrected and uncorrected errors are determined for condition (iii). Additionally, the time difference between (ii) and (iii) is calculated [74]. In this study the German paper-pencil version of the Colour-Word-Interference Test will be used (Farbe-Wort-Interferenztest, [75]).

Trail Making Test (part A and B) The Trail Making Test (TMT) is a neuropsychological test of visual search, processing speed and cognitive flexibility [76]. It consists of two tasks $\mathrm{A}$ and $\mathrm{B}$, each requiring to connect 25 consecutive targets as quickly and accurately as possible. In part A, numbers $(1-25)$ presented randomly on a piece of paper have to be connected in ascending order as fast as possible. Part $B$ requires mental shifting between presented numbers (1-13) and letters (A-L). They have to be connected in alternating order $(1, \mathrm{~A}, 2, \mathrm{~B} . .$.$) . The pro-$ cessing time for each part will be recorded.

\section{Psychosocial aspects}

Tampa Scale of Kinesiophobia The Tampa Scale of Kinesiophobia - German version (TSK-GV) is a valid and reliable 11-item self-report questionnaire measuring the fear of movement and reinjury [77]. Participants are asked to rate each item between one (strongly disagree) and four (strongly agree). Hence, a higher overall score represents a stronger individual's fear of movement [77].

Coping Strategies Questionnaire The Coping Strategies Questionnaire is a reliable measure for pain coping techniques [78]. In this study, the German version will be used (CSQ-D) [79]. The CSQ-D comprises 50 items in total. The first 48 items describe different cognitive or behavioural coping techniques (cognitive: diverting attention, reinterpreting pain sensations, coping self- 
statements, ignoring pain sensations, praying or hoping, catastrophizing; behavioural: increasing activity level, increasing pain behaviours). Participants are asked to rate how frequently they use each of the described coping techniques on a 7 -point scale $(0=$ never, $6=$ always $)$. Additionally, two items assess their self-reported overall effectiveness of their strategies. To evaluate the CSQ-D, a score is calculated for each coping technique ranging from 0 to 36 [79].

\section{Additional measurements}

Additionally, demographic (e.g. age, sex, education), physical characteristics (e.g. height, weight) and the current pain intensity on a NRS ( 0 - no pain to 10 - pain as bad as it could be) will be assessed. Moreover, information which could possibly influence the treatment outcome will be recorded. Hence, the specific characteristics and treatment history of chronic pain will be evaluated with parts of the German Pain Questionnaire $[80,81]$. The level of everyday physical activity will be assessed with the Freiburger Questionnaire on Physical Activity, collecting self-assessed data to the amount of leisure and work related activity [82, 83]. The level of state fatigue and depressive symptoms at the measurement days will be estimated trough the fatigue subscale of the Profile of Mood States (POMS-F) [84] and the Beck Depression Inventory II (BDI-II) [85].

The participants will receive a medical check-up at the end of the study period at the same orthopaedic department where the initial check-up takes place, to recognize beneficial and adverse events related to the study intervention. Any other harms, occurring during the study intervention, will be protocolled by the accredited instructors.

\section{Study procedure}

Interested subjects who will become aware of the study through the provided information (described above) can contact the research assistance of the Klinikum Magdeburg gGmbH via telephone or e-mail (see Fig. 1). Subsequently, a first eligibility check will be performed by telephone. An enrolment list will be created and constantly updated by the research assistance. Potentially eligible patients will be referred to the ambulatory health care centre of the Klinikum Magdeburg gGmbH to be informed in detail and to obtain written consent. Additionally, a medical interview will be conducted, to assess the pain characteristics and medical eligibility criteria. Finally, patients will be given a questionnaire catalogue (demographic and physical characteristics, German Pain Questionnaire, the Freiburger Questionnaire on Physical Activity, TSK-GV and CSQ-D) advising that this catalogue should be completed shortly before the pre-assessment. Subsequently, all necessary information will be forwarded to the OvGU and the participants will be invited to the pre-measurement. Two days before, they will be reminded via telephone to bring along the completed questionnaire.

Each of the measurement appointments will take about $2.5 \mathrm{~h}$. At the beginning participants will complete a set of questionnaires, including the ODI, EQ-5D-5L, POMS-F and BDI-II. Afterwards, the Colour-WordInterference Test will be performed, followed by the flexibility assessment, the TUG, the FRSTS, the dual tasks with IMUs and fNIRS, the TMTA and B and finally the $6 \mathrm{MWT}$. The group allocation will be concealed until the baseline data collection is completed. For this purpose, the enrolment list will be transferred to the OvGU, where BK will perform the group assignment based on the allocation sequence generated by MB. All participants who fulfil the inclusion criteria and give consent, will be randomly assigned to either the IG or CG group with a 1:1 allocation. Block randomization with a block size of ten will be used and the allocation is generated via a random number table. Participants will be informed by phone about their group assignment. To promote participant retention and completion of all measurements, participants will receive a financial compensation at the follow-up data collection. Moreover, the results of this study will be published in peer-reviewed journals and will be communicated with the patients and partners.

\section{Data analysis and management}

Data will be analysed using IBM SPSS 26.0 (SPSS Inc., Chicago, IL). At first, a descriptive analysis will be conducted to identify outliers, distributions and to display the baseline characteristics of both groups (means, proportions, standard deviations). To evaluate the effects of the intervention, data of all patients will be processed, regardless of whether they fully received or dropped out of the intervention (intention-to-treat-analysis). Thereby, data will be analysed until the date of drop out. As a secondary analysis, only the data from the participants who participated at least $80 \%$ of the intervention will be included in a per-protocol analysis. Missing data will be imputed using multiple imputation, under the assumption that data are missing at random. Statistical significance level will be set at $p \leq 0.05$ (1-sided).

In order to analyse differences between the groups at the time points post and follow-up, two ANCOVAs with baseline adjustments will be undertaken (covariates: baseline values and, when required, e.g. age, sex etc.) for all outcome measures. Subsequently, alpha-adjustments will be undertaken. In case the assumptions for the ANCOVA will be violated, mixed models will be used. To further elaborate the outcomes and their interactions, additional parametric or nonparametric statistical 
analyses will be applied. Exploratory mediation analysis will be performed supplementary.

To preserve the confidentiality, all participants will receive a specific code with which the data will be labelled, while the participants contact data will remain under lock. The collected data will be stored digitally and can only be accessed by the study group. Therefore, the data will be transmitted by one person and checked for correctness by another (transmission errors and plausibility). In this study, no data monitoring committee and interim analysis will be established, because of the minimal risks associated with the intervention. Overall, the data management will be in line with of the European General Data Protection Regulation.

\section{Discussion}

To the best of our knowledge, MultiMove is the first study combining motor-cognitive with dancetherapeutic as well as strength and flexibility training to address the multifaceted impairments of CLBP. First of all, it is expected that the combination of these three components will stimulate the participants in moving their spine constantly and therefore counteract the fear of moving. The group-setting will additionally provide the opportunity to experience mutual support and encouragement from peers in the engagement for physical activities [38].

Furthermore, the hypothesised advantage of MultiMove for CLBP patients is seen in the intertwining effects of the three training components. The basic component with its specific strengthening and flexibility exercises targets the whole lumbar spine including the lumbar multifidus and transversus abdominus muscles, which are known to promote the pain situation in CLPB patients [86]. The assumed enhanced lumbar stability could counteract the degeneration processes of the vertebrae and joint structures and therefore build the foundation for functional performances required during everyday activities and addressed in the motor-cognitive and dance-therapeutic component of MultiMove. Moreover, most of the movements performed in daily life are accompanied by a second task (e.g. walking and talking). This aspect will be targeted during the motor-cognitive training, where the patients will be confronted with constantly novel exercises requiring interactions between cognitive and physical performance. This ability, learned in a playful setting, might be especially valuable in enhancing the dual task performance of the patients. The following dance-therapeutic intervention stresses the mentioned aspects, as it addresses the trunk range of motion and incorporates cognitive tasks. Although, it has been shown that a dancing program is suitable to improve the motor-cognitive dual task capability in elderly [41], this study did not investigate brain activity during dual task walking. Consequently, a major advantage of this study can be seen in the methodological approach. Due to the combination of neuropsychological tests, gait analysis and fNIRS, a detailed elaboration of the respective influences on the motor-cognitive performance will be possible. These results will contribute to a better understanding which of these entities have to be addressed to reduce the impairments of CLBP patients. Furthermore, the randomized controlled design with the additional follow-up measurement will enable high level evidence on the long-term effects of MultiMove. Finally, due to the aspect that MultiMove does not need special requirements, it could be delivered on low cost in all areas, where it is needed.

One limitation in the application of MultiMove is that the participants must be physically able to participate actively in the intervention over $60 \mathrm{~min}$. Hence, CLBP patients with severe physical impairments cannot be included.

The presented study is only the first stage of a three staged project. In the second stage, it is intended to test an adapted multimodal treatment during inpatient rehabilitation and in the third stage, the programme will be integrated into the medical prevention. The overall objective is to establish the multimodal treatment into cross-sectoral care to sustainably improve the situation of CLBP patients along the entire medical supply chain.

\section{Abbreviations}

BDI-II: Beck Depression Inventory II; CES: Closed eyes standing; CG: Control group; CLBP: Chronic low back pain; CSQ-D: Coping Strategies Questionnaire - German Version; DSL: Double step length; DTS: Dual task standing; DTW: Dual task walking; FRSTST: Five-repetition sit-to-stand test; fNIRS: Functional near-infrared spectroscopy; ICD-10: International Classification of Diseases 10th revision; IG: Intervention group; IMUs: Inertial measurement units; IRB: Institutional review board; LBP: Low back pain; MTC: Minimum toe clearance; MultiMove: Multimodal intervention; NRS: Numerical rating scale; ODI: Oswestry Disability Index; OvGU: Otto von Guericke University; POMS-F: Profile of Mood States- fatigue subscale; PFC: Prefrontal cortex; STW: Single task walking; TMT: Trail Making Test; TSKGV: Tampa Scale of Kinesiophobia - German Version; TUG: Timed Up-and-Go; 6MWT: 6-min walk test

\section{Acknowledgements}

Not applicable.

\section{Protocol amendments}

If major modifications of the study protocol are necessary, all relevant institutions (e.g. IRB, funder) will be informed.

\section{Authors' contributions}

LS and JF are responsible for the project administration and provided the concept idea of the whole project. LS supervises the project and designed the study. LS and BK drafted the manuscript. All authors additionally refined the design and methodology. KCB and MB substantially contributed to the selection and adjustments of the outcome measures and edited the respective sections in the manuscript. RB developed the intervention and edited the respective section in the manuscript. $\mathrm{RB}$ and $\mathrm{MB}$ calculated the sample size and edited the respective sections in the manuscript. BK and MB planned the data management, designed the data analysis and edited the respective sections. KM and SD refined the eligibility criteria and planned the enrolment process. All authors read, revised, edited and approved the final version of the manuscript. 


\section{Funding}

The study is part of the MultiMove Project (number ZS/2019/10/101921), which is funded by the European Regional Development Fund (ERDF, project code: ajdx9exld4 EFRE 11.000sz00.00.0. 19133780 0). The funding body will not interfere in any part of the study. Open Access funding enabled and organized by Projekt DEAL.

\section{Availability of data and materials}

Not applicable.

\section{Ethics approval and consent to participate}

The study has been approved by the IRB of the Medical Faculty of the OvGU Magdeburg (No.: 182/18). A written informed consent will be obtained from each study participant.

\section{Consent for publication}

Not applicable.

\section{Competing interests}

The authors declare that they have no competing interests.

\section{Author details}

${ }^{1}$ Health and Physical Activity, Department of Sport Science, Institute III, Otto von Guericke University Magdeburg, Zschokkestraße 32, 39104 Magdeburg, Germany. ${ }^{2}$ Department of Orthopaedic Surgery, Klinikum Magdeburg gGmbH, Birkenallee 34, 39130 Magdeburg, Germany.

\section{Received: 2 October 2020 Accepted: 16 February 2021}

\section{Published online: 02 March 2021}

\section{References}

1. Schmidt CO, Raspe H, Pfingsten M, Hasenbring M, Basler HD, Eich W, Kohlmann T. Back pain in the German adult population: prevalence, severity, and sociodemographic correlates in a multiregional survey. Spine. 2007;32: 2005-11. https://doi.org/10.1097/BRS.0b013e318133fad8.

2. Hoy D, March L, Brooks P, Blyth F, Woolf A, Bain C, et al. The global burden of low back pain: estimates from the global burden of disease 2010 study. Ann Rheum Dis. 2014;73:968-74. https://doi.org/10.1136/annrheumdis-2 013-204428.

3. Airaksinen O, Brox Jl, Cedraschi C, Hildebrandt J, Klaber-Moffett J, Kovacs F, et al. Chapter 4. European guidelines for the management of chronic nonspecific low back pain. Eur Spine J. 2006;15 Suppl 2:192-300. doi:https:// doi.org/10.1007/s00586-006-1072-1.

4. Koes BW, van Tulder MW, Thomas S. Diagnosis and treatment of low back pain. BMJ. 2006:332:1430-4. https://doi.org/10.1136/bmj.332.7555.1430.

5. Adams MA. Biomechanics of back pain. Acupunct Med. 2004;22:178-88. https://doi.org/10.1136/aim.22.4.178

6. Raspe H-H. Rückenschmerzen. Berlin: Robert-Koch-Inst; 2012.

7. Lambeek LC, van Tulder MW, Swinkels ICS, Koppes LLJ, Anema JR, van Mechelen $\mathrm{W}$. The trend in total cost of back pain in the Netherlands in the period 2002 to 2007. Spine. 2011;36:1050-8. https://doi.org/10.1097/BRS. Ob013e3181e70488.

8. Juniper M, Le TK, Mladsi D. The epidemiology, economic burden, and pharmacological treatment of chronic low back pain in France, Germany, Italy, Spain and the UK: a literature-based review. Expert Opin Pharmacother. 2009;10:2581-92. https://doi.org/10.1517/14 656560903304063.

9. Karppinen J, Shen FH, Luk KDK, Andersson GBJ, Cheung KMC, Samartzis D. Management of degenerative disk disease and chronic low back pain. Orthop Clin North Am. 2011;42:513-28. https://doi.org/10.1016/j.ocl.2011. 07.009.

10. Nachemson A. Back pain. Int J Law Psychiatry. 1999;22:473-90. https://doi. org/10.1016/S0160-2527(99)00022-9.

11. Hartvigsen J, Hancock MJ, Kongsted A, Louw Q, Ferreira ML, Genevay S, et al. What low back pain is and why we need to pay attention. Lancet. 2018;391:2356-67. https://doi.org/10.1016/S0140-6736(18)30480-X.

12. Phillips FM, Slosar PJ, Youssef JA, Andersson G, Papatheofanis F. Lumbar spine fusion for chronic low back pain due to degenerative disc disease: a systematic review. Spine. 2013;38:E409-22. https://doi.org/10.1097/BRS. ob013e3182877f11.
13. Kato S, Murakami H, Demura S, Yoshioka K, Shinmura K, Yokogawa N, et al. Abdominal trunk muscle weakness and its association with chronic low back pain and risk of falling in older women. BMC Musculoskelet Disord. 2019:20:273. https://doi.org/10.1186/s12891-019-2655-4.

14. Hamacher D, Hamacher D, Schega L. A cognitive dual task affects gait variability in patients suffering from chronic low back pain. Exp Brain Res. 2014;232:3509-13. https://doi.org/10.1007/s00221-014-4039-1.

15. Brumagne $S$, Janssens $L$, Janssens $E$, Goddyn L. Altered postural control in anticipation of postural instability in persons with recurrent low back pain. Gait Posture. 2008;28:657-62. https://doi.org/10.1016/j.gaitpost.2008.04.015.

16. Monticone M, Ambrosini E, Rocca B, Magni S, Brivio F, Ferrante S. A multidisciplinary rehabilitation programme improves disability, kinesiophobia and walking ability in subjects with chronic low back pain: results of a randomised controlled pilot study. Eur Spine J. 2014;23:2105-13. https://doi.org/10.1007/s00586-014-3478-5.

17. Koch C, Hänsel F. Chronic non-specific low Back pain and motor control during gait. Front Psychol. 2018;9:2236. https://doi.org/10.3389/fpsyg.2018. 02236.

18. Hamacher D, Hamacher D, Herold F, Schega L. Are there differences in the dual-task walking variability of minimum toe clearance in chronic low back pain patients and healthy controls? Gait Posture. 2016;49:97-101. https://doi. org/10.1016/j.gaitpost.2016.06.026.

19. ljmker T, Lamoth CJC. Gait and cognition: the relationship between gait stability and variability with executive function in persons with and without dementia. Gait Posture. 2012;35:126-30. https://doi.org/10.1016/j.gaitpost.2 011.08.022.

20. Nadar MS, Jasem Z, Manee FS. The cognitive functions in adults with chronic pain: a comparative study. Pain Res Manag. 2016;2016:5719380. https://doi.org/10.1155/2016/5719380

21. Yogev-Seligmann G, Hausdorff JM, Giladi N. The role of executive function and attention in gait. Mov Disord. 2008;23:329-42; quiz 472. https://doi. org/10.1002/mds.21720.

22. Truchon M. Determinants of chronic disability related to low back pain: towards an integrative biopsychosocial model. Disabil Rehabil. 2001;23:75867. https://doi.org/10.1080/09638280110061744.

23. Vlaeyen JW, Kole-Snijders AM, Rotteveel AM, Ruesink R, Heuts PH. The role of fear of movement/(re)injury in pain disability. J Occup Rehabil. 1995;5: 235-52. https://doi.org/10.1007/BF02109988.

24. Vlaeyen JW, Linton SJ. Fear-avoidance and its consequences in chronic musculoskeletal pain: a state of the art. Pain. 2000;85:317-32. https://doi. org/10.1016/S0304-3959(99)00242-0.

25. Yelvar GD, Çırak Y, Dalkılınç M, Parlak Demir Y, Guner Z, Boydak A. Is physiotherapy integrated virtual walking effective on pain, function, and kinesiophobia in patients with non-specific low-back pain? Randomised controlled trial. Eur Spine J. 2017;26:538-45. https://doi.org/10.1007/s00586016-4892-7

26. Mutubuki EN, Beljon Y, Maas ET, Huygen FJPM, Ostelo RWJG, van Tulder MW, van Dongen JM. The longitudinal relationships between pain severity and disability versus health-related quality of life and costs among chronic low back pain patients. Qual Life Res. 2020;29:275-87. https://doi.org/10.1 007/s11136-019-02302-w.

27. Kamper SJ, Apeldoorn AT, Chiarotto A, Smeets RJEM, Ostelo RWJG, Guzman $J$, van Tulder MW. Multidisciplinary biopsychosocial rehabilitation for chronic low back pain: Cochrane systematic review and meta-analysis. BMJ. 2015; 350:h444. https://doi.org/10.1136/bmj.h444.

28. Guzmán J, Esmail R, Karjalainen K, Malmivaara A, Irvin E, Bombardier C. Multidisciplinary bio-psycho-social rehabilitation for chronic low back pain. Cochrane Database Syst Rev. 2002:CD000963. doi:https://doi.org/10.1002/14 651858.CD000963.

29. Pieber K, Herceg M, Csapo R, Wiesinger G, Quittan M, Crevenna R, Mittermaier C. Effects of a multidisciplinary programme on postural stability in patients with chronic recurrent low back pain: preliminary findings. Eur Spine J. 2016;25:1219-25. https://doi.org/10.1007/s00586-015-4293-3.

30. Searle A, Spink M, Ho A, Chuter V. Exercise interventions for the treatment of chronic low back pain: a systematic review and meta-analysis of randomised controlled trials. Clin Rehabil. 2015;29:1155-67. https://doi.org/1 $0.1177 / 0269215515570379$.

31. Hayden JA, van Tulder MW, Tomlinson G. Systematic review: strategies for using exercise therapy to improve outcomes in chronic low back pain. Ann Intern Med. 2005;142:776-85. https://doi.org/10.7326/0003-4819-142-9-2 00505030-00014. 
32. Lutz H. Life Kinetik ${ }^{\otimes}$ : Bewegung macht Hirn. 1st ed. Aachen: Meyer \& Meyer; 2017.

33. Barban F, Annicchiarico R, Melideo M, Federici A, Lombardi MG, Giuli S, et al. Reducing fall risk with combined motor and cognitive training in elderly fallers. Brain Sci. 2017. https://doi.org/10.3390/brainsci7020019.

34. Theill $N$, Schumacher $V$, Adelsberger R, Martin $M$, Jäncke L. Effects of simultaneously performed cognitive and physical training in older adults. BMC Neurosci. 2013;14:103. https://doi.org/10.1186/1471-2202-14-103.

35. Kattenstroth J-C, Kolankowska I, Kalisch T, Dinse HR. Superior sensory, motor, and cognitive performance in elderly individuals with multi-year dancing activities. Front Aging Neurosci. 2010;2:31. https://doi.org/10.3389/fnagi.201 0.00031 .

36. Dhami P, Moreno S, DeSouza JFX. New framework for rehabilitation - fusion of cognitive and physical rehabilitation: the hope for dancing. Front Psychol. 2014;5:1478. https://doi.org/10.3389/fpsyg.2014.01478.

37. Liu X, Shen P-L, Tsai Y-S. Dance intervention effects on physical function in healthy older adults: a systematic review and meta-analysis. Aging Clin Exp Res. 2020. https://doi.org/10.1007/s40520-019-01440-y.

38. Yan A, Cobley S, Chan C, Pappas E, Nicholson LL, Ward RE, et al. The effectiveness of dance interventions on physical health outcomes compared to other forms of physical activity: a systematic review and metaanalysis. Sports Med. 2018;48:933-51. https://doi.org/10.1007/s40279-0170853-5.

39. Kattenstroth J-C, Kalisch T, Holt S, Tegenthoff M, Dinse HR. Six months of dance intervention enhances postural, sensorimotor, and cognitive performance in elderly without affecting cardio-respiratory functions. Front Aging Neurosci. 2013;5:5. https://doi.org/10.3389/fnagi.2013.00005.

40. Bennett CG, Hackney ME. Effects of line dancing on physical function and perceived limitation in older adults with self-reported mobility limitations. Disabil Rehabil. 2018:40:1259-65. https://doi.org/10.1080/09638288.2017.12 94207.

41. Hamacher D, Hamacher D, Rehfeld K, Hökelmann A, Schega L. The effect of a six-month dancing program on motor-cognitive dual-task performance in older adults. J Aging Phys Act. 2015;23:647-52. https://doi.org/10.1123/ja pa.2014-0067.

42. Rehfeld K, Lüders A, Hökelmann A, Lessmann V, Kaufmann J, Brigadski T, et al. Dance training is superior to repetitive physical exercise in inducing brain plasticity in the elderly. PLoS One. 2018;13:e0196636. https://doi.org/1 0.1371/journal.pone.0196636.

43. Bearss KA, McDonald KC, Bar RJ, DeSouza JF. Improvements in balance and gait speed after a 12 week dance intervention for Parkinson's disease. Advances in Integrative Medicine. 2017:4:10-3. https://doi.org/10.1016/j.a imed.2017.02.002.

44. Krishnamurthi N, Murphey C, Driver-Dunckley E. A comprehensive movement and motion training program improves mobility in Parkinson's disease. Aging Clin Exp Res. 2020;32:633-43. https://doi.org/10.1007/s40520019-01236-0.

45. Ruiz-Muelle A, López-Rodríguez MM. Dance for people with Alzheimer's disease: a systematic review. Curr Alzheimer Res. 2019;16:919-33. https://doi. org/10.2174/1567205016666190725151614.

46. Ho RTH, Fong TCT, Chan WC, Kwan JSK, Chiu PKC, Yau JCY, Lam LCW. Psychophysiological effects of dance movement therapy and physical exercise on older adults with mild dementia: a randomized controlled trial. J Gerontol B Psychol Sci Soc Sci. 2020;75:560-70. https://doi.org/10.1093/ geronb/gby 145 .

47. Lim C-Y, In J. Randomization in clinical studies. Korean J Anesthesiol. 2019; 72:221-32. https://doi.org/10.4097/kja.19049.

48. Janda V. Manuelle Muskelfunktionsdiagnostik. 4th ed. München: Elsevier Urban \& Fischer; 2009

49. Britten L, Addington C, Astill S. Dancing in time: feasibility and acceptability of a contemporary dance programme to modify risk factors for falling in community dwelling older adults. BMC Geriatr. 2017;17:83. https://doi.org/1 0.1186/s12877-017-0476-6.

50. Granacher U, Lacroix A, Muehlbauer T, Roettger K, Gollhofer A. Effects of core instability strength training on trunk muscle strength, spinal mobility, dynamic balance and functional mobility in older adults. Gerontology. 2013; 59:105-13. https://doi.org/10.1159/000343152.

51. Vaughan S, Wallis M, Polit D, Steele M, Shum D, Morris N. The effects of multimodal exercise on cognitive and physical functioning and brainderived neurotrophic factor in older women: a randomised controlled trial. Age Ageing. 2014;43:623-9. https://doi.org/10.1093/ageing/afu010.
52. Garber CE, Blissmer B, Deschenes MR, Franklin BA, Lamonte MJ, Lee I-M, et al. American College of Sports Medicine position stand. Quantity and quality of exercise for developing and maintaining cardiorespiratory, musculoskeletal, and neuromotor fitness in apparently healthy adults: guidance for prescribing exercise. Med Sci Sports Exerc. 2011;43:1334-59. https://doi.org/10.1249/MSS.0b013e318213fefb.

53. Wendel I, Patel SI, Wyss J. Home exercise programs for musculoskeletal and sports injuries: the evidence-based guide for practitioners: springer publishing company; 2019

54. Jekauc D, Voelkle M, Wagner MO, Mewes N, Woll A. Reliability, validity, and measurement invariance of the German version of the physical activity enjoyment scale. J Pediatr Psychol. 2013;38:104-15. https://doi.org/10.1093/ jpepsy/jss088.

55. Herman T, Giladi N, Hausdorff JM. Properties of the 'timed up and go' test: more than meets the eye. Gerontology. 2011;57:203-10. https://doi.org/1 $0.1159 / 000314963$

56. Podsiadlo D, Richardson S. The timed "up \& go": a test of basic functional mobility for frail elderly persons. J Am Geriatr Soc. 1991;39:142-8. https:// doi.org/10.1111/j.1532-5415.1991.tb01616.x.

57. Lin M-R, Hwang H-F, Hu M-H, Wu H-DI, Wang Y-W, Huang F-C. Psychometric comparisons of the timed up and go, one-leg stand, functional reach, and Tinetti balance measures in community-dwelling older people. J Am Geriatr Soc. 2004;52:1343-8. https://doi.org/10.1111/j.1532-541 5.2004.52366.x.

58. Enright PL. The six-minute walk test. Respir Care. 2003;48:783-5.

59. Hamacher D, Hamacher D, Taylor WR, Singh NB, Schega L. Towards clinical application: repetitive sensor position re-calibration for improved reliability of gait parameters. Gait Posture. 2014;39:1146-8. https://doi.org/10.1016/j.ga itpost.2014.01.020

60. Callisaya ML, Blizzard L, Schmidt MD, Martin KL, McGinley JL, Sanders LM, Srikanth VK. Gait, gait variability and the risk of multiple incident falls in older people: a population-based study. Age Ageing. 2011;40:481-7. https:// doi.org/10.1093/ageing/afr055.

61. Micklewright D. St Clair Gibson a, Gladwell V, Al Salman a. development and validity of the rating-of-fatigue scale. Sports Med. 2017;47:2375-93. https:// doi.org/10.1007/s40279-017-0711-5.

62. Bohannon RW, Bubela DJ, Magasi SR, Wang Y-C, Gershon RC. Sit-to-stand test: performance and determinants across the age-span. Isokinet Exerc Sci. 2010;18:235-40. https://doi.org/10.3233/IES-2010-0389.

63. Simmonds MJ, Olson SL, Jones S, Hussein T, Lee CE, Novy D, Radwan H. Psychometric characteristics and clinical usefulness of physical performance tests in patients with low back pain. Spine. 1998;23:2412-21. https://doi. org/10.1097/00007632-199811150-00011.

64. Fairbank JC, Couper J, Davies JB, O'Brien JP. The Oswestry low back pain disability questionnaire. Physiotherapy. 1980;66:271-3.

65. Fairbank JC, Pynsent PB. The Oswestry disability index. Spine. 2000;25:294052; discussion 2952. https://doi.org/10.1097/00007632-200011150-00017.

66. Mannion AF, Junge A, Fairbank JCT, Dvorak J, Grob D. Development of a German version of the Oswestry disability index. Part 1: cross-cultural adaptation, reliability, and validity. Eur Spine J. 2006;15:55-65. https://doi. org/10.1007/s00586-004-0815-0

67. Herdman M, Gudex C, Lloyd A, Janssen M, Kind P, Parkin D, et al. Development and preliminary testing of the new five-level version of EQ-5D (EQ-5D-5L). Qual Life Res. 2011;20:1727-36. https://doi.org/10.1007/s11136011-9903-X.

68. Ludwig K. Graf von der Schulenburg J-M, Greiner W. German Value Set for the EQ-5D-5L. Pharmacoeconomics. 2018;36:663-74. https://doi.org/10.1007/ s40273-018-0615-8.

69. Menant JC, Maidan I, Alcock L, Al-Yahya E, Cerasa A, Clark DJ, et al. A consensus guide to using functional near-infrared spectroscopy in posture and gait research. Gait Posture. 2020;82:254-65. https://doi.org/10.1016/j.ga itpost.2020.09.012

70. Scholkmann F, Kleiser S, Metz AJ, Zimmermann R, Mata Pavia J, Wolf U, Wolf $M$. A review on continuous wave functional near-infrared spectroscopy and imaging instrumentation and methodology. Neuroimage. 2014;85(Pt 1):627. https://doi.org/10.1016/..neuroimage.2013.05.004.

71. Stroop JR. Studies of interference in serial verbal reactions. J Exp Psychol. 1935;18:643-62. https://doi.org/10.1037/h0054651.

72. Diamond A. Executive functions. Annu Rev Psychol. 2013;64:135-68. https:// doi.org/10.1146/annurev-psych-113011-143750. 
73. Scarpina F, Tagini S. The Stroop color and word test. Front Psychol. 2017;8: 557. https://doi.org/10.3389/fpsyg.2017.00557.

74. Uttl B, Graf P. Color-word Stroop test performance across the adult life span. J Clin Exp Neuropsychol. 1997;19:405-20. https://doi.org/10.1080/0168863 9708403869

75. Bäumler G. Farbe-Wort-Interferenztest (FWIT) nach JR Stroop: Handanweisung. München: Verlag für Psychologie Hogrefe; 1985.

76. Tombaugh T. Trail making test a and B: normative data stratified by age and education. Arch Clin Neuropsychol. 2004;19:203-14. https://doi.org/10.1 016/50887-6177(03)00039-8.

77. Rusu AC, Kreddig N, Hallner D, Hülsebusch J, Hasenbring MI. Fear of movement/(re)injury in low back pain: confirmatory validation of a German version of the Tampa scale for Kinesiophobia. BMC Musculoskelet Disord. 2014;15:280. https://doi.org/10.1186/1471-2474-15-280.

78. Rosenstiel AK, Keefe FJ. The use of coping strategies in chronic low back pain patients: relationship to patient characteristics and current adjustment. Pain. 1983;17:33-44. https://doi.org/10.1016/0304-3959(83)90125-2.

79. Verra ML, Angst F, Lehmann S, Aeschlimann A. Translation, cross-cultural adaptation, reliability, and validity of the German version of the coping strategies questionnaire (CSQ-D). J Pain. 2006;7:327-36. https://doi.org/10.1 016/j.jpain.2005.12.005.

80. Nagel B, Pfingsten M, Lindena G, Kohlmann T. Handbuch Deutscher Schmerzfragebogen. 1st ed. Berlin; 2015

81. Nagel B, Gerbershagen HU, Lindena G, Pfingsten M. Entwicklung und empirische Uberprüfung des Deutschen Schmerzfragebogens der DGSS Schmerz. 2002;16:263-70. https://doi.org/10.1007/s00482-002-0162-1.

82. Frey I, Berg A, Grathwohl D, Keul J. Freiburger Fragebogen zur körperlichen Aktivität-Entwicklung, Prüfung und Anwendung. Soz Praventivmed. 1999; 44:55-64. https://doi.org/10.1007/BF01667127.

83. Frey I, Berg A. Physical activity counseling: assessment of physical activity by questionnaire. Eur J Sport Sci. 2002;2:1-6. https://doi.org/10.1080/174613902 00072406.

84. McNair D, Lorr M, Droppleman L. Manual for Profile of Mood States. Educational and Industrial Testing Services. 1992.

85. Hautzinger M, Keller F, Kühner C. Beck depressions-Inventar (BDI-II). Frankfurt/Main: Harcourt Test Services; 2006.

86. Russo M, Deckers K, Eldabe S, Kiesel K, Gilligan C, Vieceli J, Crosby P. Muscle control and non-specific chronic low Back pain. Neuromodulation. 2018;21: 1-9. https://doi.org/10.1111/ner.12738.

\section{Publisher's Note}

Springer Nature remains neutral with regard to jurisdictional claims in published maps and institutional affiliations.

Ready to submit your research? Choose BMC and benefit from:

- fast, convenient online submission

- thorough peer review by experienced researchers in your field

- rapid publication on acceptance

- support for research data, including large and complex data types

- gold Open Access which fosters wider collaboration and increased citations

- maximum visibility for your research: over $100 \mathrm{M}$ website views per year

At BMC, research is always in progress.

Learn more biomedcentral.com/submissions 